\title{
Social subordination alters estradiol-induced changes in cortico-limbic brain volumes in adult female rhesus monkeys
}

\author{
Katherine M. Reding ${ }^{\mathrm{a}}$, Martin M. Styner ${ }^{\mathrm{b}}$, Mark E. Wilson ${ }^{\mathrm{a}, \mathrm{d}}$, Donna Toufexis ${ }^{\mathrm{c}}$, \\ Mar M. Sanchez ${ }^{\mathrm{a}, \mathrm{d}, *}$ \\ ${ }^{a}$ Yerkes National Primate Research Center, Emory University, United States \\ ${ }^{\mathrm{b}}$ Department of Psychiatry, University of North Carolina - Chapel Hill, United States \\ ${ }^{c}$ Department of Psychological Science, University of Vermont, United States \\ ${ }^{\mathrm{d}}$ Department of Psychiatry \& Behavioral Sciences, Emory University, United States
}

\section{A R T I C L E I N F O}

Keywords:

Rhesus monkey

SMRI

Estradiol

Stress

\begin{abstract}
A B S T R A C T
Women have a higher risk of developing stress-related disorders compared to men and the experience of a stressful life event is a potent risk-factor. The rodent literature suggests that chronic exposure to stressors as well as 17ß-estradiol (E2) can result in alterations in neuronal structure in corticolimbic brain regions, however the translation of these data to humans is limited by the nature of the stressor experienced and issues of brain homology. To address these limitations, we used a well-validated rhesus monkey model of social subordination to examine effects of E2 treatment on subordinate (high stress) and dominant (low stress) female brain structure, including regional gray matter and white matter volumes using structural magnetic resonance imaging. Our results show that one month of E2 treatment in ovariectomized females, compared to control (no) treatment, decreased frontal cortex gray matter volume regardless of social status. In contrast, in the cingulate cortex, an area associated with stress-induced emotional processing, E2 decreased grey matter volume in subordinates but increased it in dominant females. Together these data suggest that physiologically relevant levels of E2 alter cortical gray matter volumes in females after only one month of treatment and interact with chronic social stress to modulate these effects on brain structure.
\end{abstract}

\section{Introduction}

Women are two times more vulnerable to develop stress-related psychological disorders than men (Kessler, 2003), and the experience of stressful life events often precipitates impaired emotional regulation and emergence of mood and anxiety disorders and other adverse health outcomes (Kendler et al., 1993). One possibility for these sex differences is an interaction between chronic stress and ovarian hormone signaling, such that $17 \beta$-estradiol (E2) may exacerbate the negative effects of stress hormones, such as glucocorticoids (GCs), in women (Seeman, 1997). Corticolimbic regions involved in socioemotional processing, including the prefrontal cortex (PFC), cingulate cortex, amygdala, and hippocampus, express high levels of both GC and estrogen receptors (Morimoto et al., 1996; Sánchez et al., 2000; Shughrue et al., 1997), making them sensitive to effects of stress and E2. A better understanding of how chronic stress interacts with E2 to modify these structures may provide insight into the etiology of and treatment for stress-related disorders in women.

Prolonged stress has been shown to have damaging effects on rodent corticolimbic brain regions including the PFC, cingulate cortex, amygdala, and hippocampus (Joels et al., 2007). In the hippocampus, chronic stress or GC exposure reduces neuronal dendritic arborization and length as well as spine density (McEwen, 1999). Chronic stress also decreases dendritic arborization in the medial (m)PFC (Radley et al., 2013) while it has opposite effects on the amygdala, increasing dendritic arborization (Rosenkranz et al., 2010). These structural alterations are associated with increased fear and anxiety-like behavior. However, these studies focused mainly on males and may not be translatable to females. Although ovariectomized females without E2 treatment show stress-related dendritic retraction in hippocampal neurons similar to males (McEwen, 1999; McLaughlin et al., 2010), they do not show the stress effects reported in male PFC (Garrett and Wellman, 2009). Indeed, in ovariectomized females receiving E2 treatment, chronic restraint stress results in the opposite: increased

\footnotetext{
${ }^{*}$ Corresponding author at: Department of Psychiatry \& Behavioral Sciences, School of Medicine, Core Scientist, Yerkes National Primate Research Center, Emory University, 954 Gatewood Rd NE, Atlanta, GA 30329, United States.

E-mail address: mmsanch@emory.edu (M.M. Sanchez).
} 
apical dendritic length (Garrett and Wellman, 2009) and spine density in $\mathrm{mPFC}$ neurons projecting to the amygdala (Shansky et al., 2010). Structural effects are seen even in the absence of stress, as mPFC neurons show dendritic retraction following ovariectomy (Wallace et al., 2006) and growth following E2 (Shansky et al., 2010), suggesting that chronic restraint stress could act to potentiate E2's neurotrophic effects in the female rodent mPFC. Stress and E2 have a different interaction effect in the hippocampus, such that E2 protects against stress-related atrophy, increases spine density, and facilitates hippocampal-dependent cognitive tasks (e.g. Morris water maze) (McLaughlin et al., 2010). This is consistent with a large body of literature supporting E2-dependent neurotrophic effects in hippocampal neurons, although sometimes region-specific (Gould et al., 1990), and it suggests that chronic stress acts to modify E2's trophic effects in the rodent hippocampus. In the amygdala, elevated levels of E2 and progesterone during proestrus have been associated with decreased spine density (Rubinow et al., 2009) although the specific interaction of stress and E2 was not examined. Overall, the literature on the interaction of stress and E2 on brain structure has been sparse, limited to rodent models, and using chronic stress paradigms that are difficult to relate to human females.

Our understanding of the neurobiological effects of stress and E2 on human brain structure is limited by several methodological factors. First, experimental manipulation of exposure to chronic stress is not possible in humans. Data from adult individuals with stress-related psychopathology are confounded by both experience of early life stress and comorbid clinical features of the disease (e.g., post-traumatic stress disorder, depression). Second, to determine the specific effects of E2, experimental manipulation of endogenous hormones is necessary and involves either chemical ovarian suppression or ovariectomy. Third, examination of structural changes (dendritic arborization, length, and spine density) via post-mortem histological analyses is not feasible for conducting randomized studies of either stress or E2 exposure. However, non-invasive measures of gray and white matter volume by structural magnetic resonance imaging (sMRI) are commonly used and thought to reflect alterations in dendritic arborization, length, and synaptic density (Kassem et al., 2013). To address these limitations, we chose to use a rhesus monkey model to investigate the specific impact of chronic psychosocial stress and its interactions with E2 on brain structure using sMRI. Social subordination in adult female macaques is a well-established and highly translational animal model used to examine the consequences of chronic exposure to psychosocial stress in women (Michopoulos et al., 2012a; Wilson, 2016). Subordinate social status in female rhesus monkeys results in myriad stress-related phenotypes including reduced rates of prosocial behaviors (Michopoulos et al., 2012a; Reding et al., 2012), a dysregulated stress axis (Michopoulos et al., 2012a; Michopoulos et al., 2012b), reduced neurotrophin levels (Michopoulos et al., 2012a), stress-induced overeating, and obesity (Godfrey et al., 2018; Michopoulos et al., 2012c; Shively et al., 2009). Furthermore, the use of ovariectomized monkeys allows us to experimentally control exposure to E2 and stress and examine the effects of both, either alone or combined, on the female primate brain. Thus, by using the rhesus monkey model, we can examine the interaction of chronic stress and E2 on brain structure in an animal model more closely related to human females.

The aim of the current study is to identify the specific structural impact of social subordination (stress) and E2 (with or without stress) on hippocampus and amygdala and both gray and white matter volumes in the PFC, cingulate, and frontal cortices. We hypothesized that subordinate social status (high stress) would result in reduced hippocampal, PFC, frontal, and cingulate volumes, but in increased amygdala volume (at least based on the existing reports in males). Furthermore, we hypothesized that E2 treatment would increase cortical and hippocampal volumes and decrease amygdala volumes. Lastly, we hypothesized that E2 replacement in combination with chronic stress would be protective because of the neurotrophic effects reported in cortical and hippocampal brain regions, but detrimental in the amygdala. Understanding whether stress attenuates or exacerbates E2's structural alterations in primate corticolimbic regions can increase our understanding of its potential role on increased vulnerability to stressrelated pathology and disorders such as anxiety and depression in adult women.

\section{Methods}

\subsection{Subjects}

Subjects were 20 ovariectomized adult female rhesus monkeys (Macaca mulatta), socially-housed and maintained at the Yerkes National Primate Research Center (YNPRC) Field Station. Subjects were classified by social status, either the most dominant (DOM, Rank 1, N = 10 ) or the most subordinate (SUB, Rank 4 or $5, N=10$ ) females in each of ten groups of 4-5 females housed with one resident adult male. Subjects were housed in indoor - outdoor enclosures that measured 20 $\times 15 \times 8$ feet each. Animals were fed a standard commercial low-fat high-fiber monkey chow diet (Ralston Purina Company, St. Louis MO) ad libitum supplemented daily with seasonal fruits and vegetables. All procedures were approved by the Emory University Institutional Animal Care and Use Committee (IACUC) in accordance with the Animal Welfare Act and the U.S. Department of Health and Human Services "Guide for Care and Use of Laboratory Animals."

Social groups containing the subjects had been established as previously described (Jarrell et al., 2008). Briefly, middle ranking, ovariectomized females were removed from their natal breeding groups ( $>100$ animals each) and introduced to one another to form new smaller groups of five unrelated, unfamiliar females and one adult male each. Females were added sequentially, and dominance hierarchies were established based largely on order of introduction, with the final female often assuming the lowest rank. Since group formation, subjects have been included in several studies in which all females received periodic replacement therapy with E2 lasting approximately 2-4 weeks and/or progesterone for similar durations (Michopoulos et al., 2009, 2011; Reding et al., 2012). During the course of data collection for this study, two DOM females were unable to complete both treatment conditions, and were therefore excluded from the analysis, bringing the sample size to 18 females. In the final sample, animals were $14( \pm 2)$ years of age with no significant age difference between groups (DOM = $14 \pm 2 ;$ SUB $\left.=14 \pm 3 ; \mathrm{F}_{1,16}=.02, \mathrm{p}=.90, \eta_{\mathrm{p}}^{2}<.01\right)$. Furthermore, all females were ovariectomized $6( \pm 2)$ years prior to the start of the study with no significant difference between DOM and SUB females $\left(\mathrm{DOM}=5 \pm 2 ;\right.$ SUB $\left.=7 \pm 2 ; \mathrm{F}_{1,16}=2.54, \mathrm{p}=.13, \eta_{\mathrm{p}}^{2}=.14\right)$. Finally, there was no difference in the age of ovariectomy between DOM $(8.1 \pm 2.3$ years $)$ and SUB females $\left(6.4 \pm 4.1\right.$ years, $\mathrm{F}_{1,16}=1.14, \mathrm{p}=$ $\left..30, \eta_{\mathrm{p}}^{2}<.07\right)$.

\subsection{Social subordination}

Rhesus monkey social groups are structured in linear dominance hierarchies that are maintained by both contact and non-contact aggressive behavior from more DOM females to more SUB females (Bernstein et al., 1974; Michopoulos et al., 2012a). Thus, frequency of aggressive, affiliative, and submissive behaviors are status dependent, with SUB females receiving the most aggression, often random and unpredictable, from more DOM animals as well as exhibiting the highest rates of submissive behaviors directed toward more DOM animals to attenuate the aggression (Silk, 2002). Previous studies from our lab demonstrated that SUB females have increased behavioral, metabolic, and neuroendocrine markers of a chronic stress phenotype in comparison to the highest-ranking DOM females. Specifically, our lab has documented that subordinate social status is a potent chronic stressor (Wilson, 2016), including in this specific group of female rhesus monkeys (Michopoulos et al., 2012a, b), based on hypothalamic-pituitary-adrenal (HPA) axis function assessments (e.g. impaired negative 

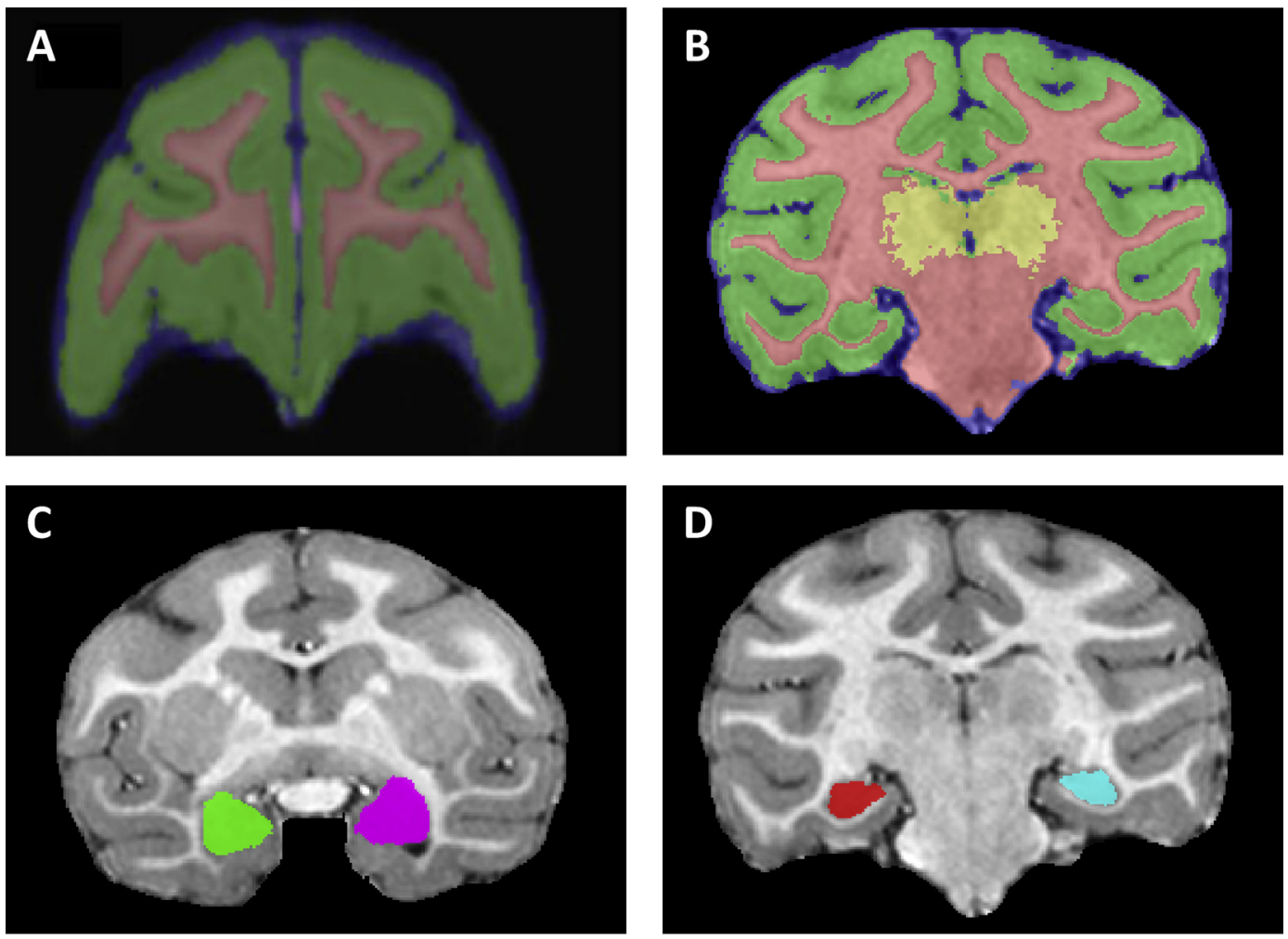

\section{$\mathbf{E}$}

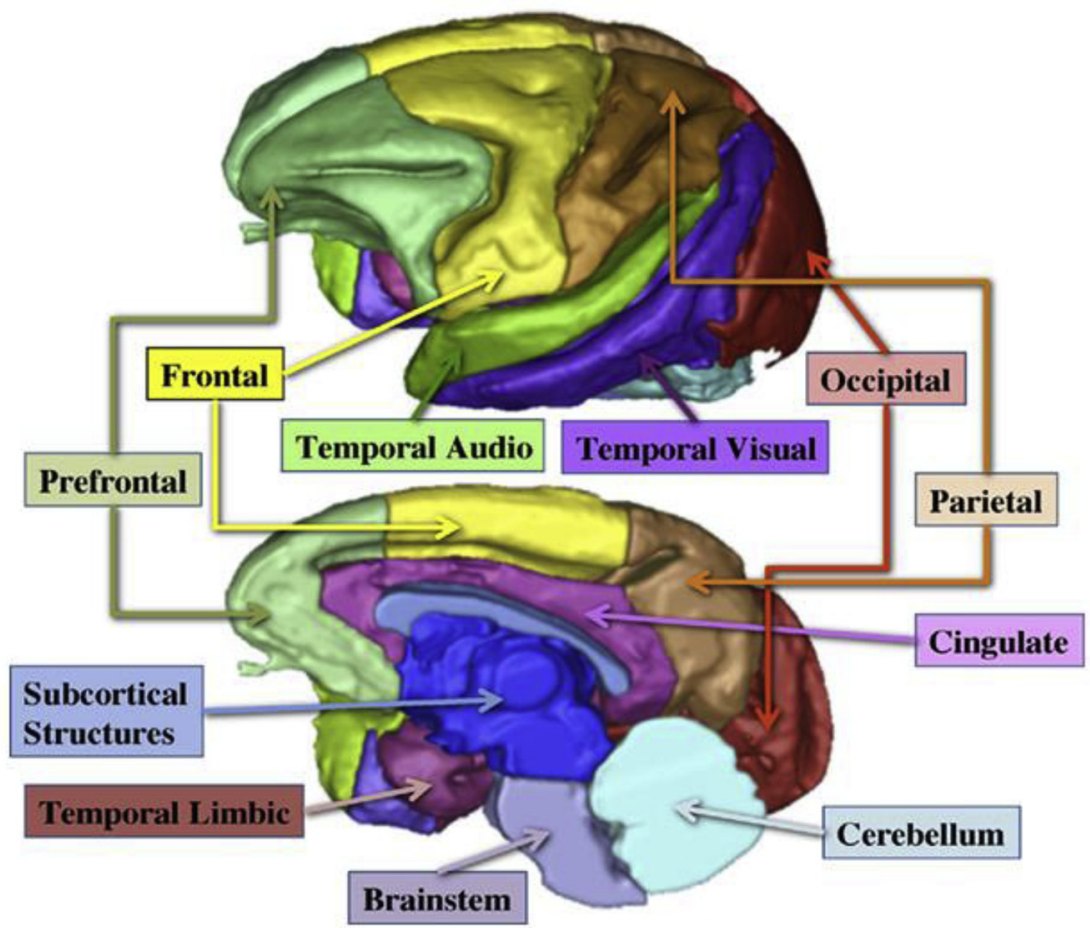

Fig. 1. Images depict the AutoSeg (version 2.6.2) automatic parcellation of brain tissue (Wang et al., 2014). (A-B) Parcellations of gray matter (green), white matter (red), CSF (blue), subcortical tissue (yellow), (C) amygdala, and (D) hippocampus were manually adjusted to ensure accurate neuroanatomical delineation. Segmentations of lobar volumes (E, image courtesy of (Short et al., 2010)) prefrontal (light green), (B) frontal (yellow), and cingulate (light purple) were used to delineate both gray and white matter within each subdivision.

feedback), and stress-related behavioral phenotypes. Thus, social subordination in female rhesus monkeys provides a well-validated translational model to further explore the consequences of exposure to chronic stressors in both non-human primates and women (Michopoulos et al., 2012a; Wilson, 2016).

\subsection{Estradiol treatment}

All females were studied during an E2 replacement and a control, no treatment, condition as previously described (Asher et al., 2013). The order of treatment was counterbalanced across female social status. In the E2 condition, treatment was administered via E2-filled continuous- 
release Silastic ${ }^{\circledast}$ capsules placed between the scapulae under anesthesia, to yield serum levels corresponding to the mid- to late follicular phase in this species (Wilson et al., 1982) for an average of 31 $( \pm 3)$ days. To verify Silastic capsule efficacy and E2 levels in both conditions, serum samples $(200 \mu \mathrm{l})$ were collected during week 2 of each condition (E2 treatment and control) and at the time of capsule removal or approximately 4-6 weeks after the start of the control condition (26). E2 levels were measured using a modification of a previously validated commercial assay (Siemens/DPC, Los Angeles, CA, USA). The assay kit has a sensitivity of $5 \mathrm{pg} / \mathrm{ml}$ and an intra- and interassay coefficient of variation (CV) of $5.2 \%$ and $11.1 \%$, respectively. During the E2 condition, there were no differences in serum levels (pg/ $\mathrm{ml})$ between DOM $(95.1 \pm 6.9)$ and SUB $\left(91.5 \pm 6.2 ; \mathrm{F}_{1,16}=0.15, \mathrm{p}=\right.$ $\left..70, \eta_{\mathrm{p}}^{2} \leq .01\right)$ nor between week 2 of treatment $(90.6 \pm 5.3)$ and just prior to capsule removal $\left(96.0 \pm 6.1 ; \mathrm{F}_{1,16}=0.64, \mathrm{p}=.44, \eta_{\mathrm{p}}^{2}=.04\right)$. In addition, there were no significant differences between DOM and SUB females in duration of time females were treated with E2 at the time of the scan (DOM $=33 \pm 12$ days; SUB $=33 \pm 11$ days; $\left.\mathrm{F}_{1,16}<.001, \mathrm{p}=.99, \eta_{\mathrm{p}}^{2}<.01\right)$. During the control condition, serum E2 was consistently below assay detectability $(<5 \mathrm{pg} / \mathrm{ml})$ in both DOMs and SUBs, and the interval of time between the previous E2 treatment and the scan (mean of $43( \pm 13)$ days) did not vary significantly based on status (DOM $=43 \pm 14$ days; SUB $=44 \pm 12$ days; $\left.\mathrm{F}_{1,16}=.07, \mathrm{p}=.78, \eta_{\mathrm{p}}^{2}<0.01\right)$.

\subsection{Neuroimaging protocol}

\subsubsection{Structural MRI acquisition}

All females were scanned twice: during the E2 treatment and the control condition, in counterbalanced order. Subjects were transported from their social group to the YNPRC MRI Center the day before the scans. All scans were acquired on a $3 \mathrm{~T}$ Siemens Magnetom TRIO system (Siemens Med. Sol., Malvern, PA, USA) and using an 8-channel phase array coil. Two structural scans (T1- and T2-weighted MRI) were conducted during each session. The T1-MRI scan was acquired using a 3dimensional (3D) magnetization-prepared rapid gradient-echo (3DMPRAGE) parallel imaging sequence (TR/TE/TI $=3000 / 3.52 \mathrm{~ms} / 950$ ms, voxel-size $=0.5 \mathrm{~mm}^{3}$, isotropic, 6 averages). A T2-weighted MR scan was collected in the same direction as the T1 (TR/TE $=7900 / 125$ $\mathrm{ms}$, voxel size $=0.5 \times 0.5 \times 1.0 \mathrm{~mm}^{3}, 10$ averages) in order to aid with delineation of regions of interest (ROIs) by improving the contrast of grey matter, white matter, and cerebrospinal fluid (CSF) borders (Knickmeyer et al., 2010). All animals were scanned supine in the same orientation, achieved by placement and immobilization of the head in a custom-made head holder via ear bars and a mouth piece. A vitamin E capsule was taped on the right temple to mark the right side of the brain. Scans were acquired under isoflurane anesthesia (1-1.2 \% to effect, inhalation), following initial induction with Telazol $(5 \mathrm{mg} / \mathrm{kg}$, i.m.). Animals were fitted with an oximeter, ECG, rectal thermistor and blood pressure monitor for physiological monitoring, an i.v. catheter to administer dextrose $/ \mathrm{NaCl}(0.45 \%)$ to maintain normal hydration and an MRI-compatible heating pad. Upon completion of the scans and full recovery from anesthesia, each female was returned to their social group.

\subsubsection{MRI data processing and analysis}

Structural data were analyzed using an automatic atlas-based segmentation program, AutoSeg (version 2.6.2), an open-source software pipeline developed at the Neuro Image Research and Analysis Laboratories of the University of North Carolina at Chapel Hill (Wang et al., 2014). AutoSeg is used to automatically parcellate brain tissue (Fig. 1A-E) including gray matter, white matter, CSF, cortical lobes, and subcortical structures in the rhesus macaque as described previously (Knickmeyer et al., 2010). Briefly, the subjects' MRI images were registered to a T1-MRI rhesus atlas image and then automatically parcellated based on the subject's T1- and T2-weighted scans into cortical lobes and subcortical regions, as well as automatically segmented into gray matter, white matter, and CSF as previously published (Styner et al., 2007). For this study, automatic parcellations and segmentations of lobar volumes (gray matter and white matter) corresponding to the PFC, cingulate, and frontal cortices (Fig. 1E), as well as the subcortical hippocampus and amygdala ROIs (Fig. 1C-D) were manually adjusted to ensure accurate neuroanatomical delineation by two raters who remained blind to experimental groups and using published anatomical criteria for the macaque brain (Paxinos et al., 2000; Saleem and Logothetis, 2006). Lobar segmentations corresponding to the PFC, cingulate, and frontal cortices were defined as follows (for more details see Fig. 1E and Knickmeyer et al., 2010, supplemental material). The PFC anterior and lateral boundaries were defined by CSF, medially by the interhemispheric fissure, and posteriorly by the arcuate sulcus. Moving anterior to posterior, the inferior boundary was initially CSF, then the sylvian fissure, and finally the arcuate sulcus. This parcellation included Brodmann's areas (BA) 8-14, 32,45 , and 46 . The frontal lobe anterior boundary was defined by the PFC, laterally by the CSF, medially by the interhemispheric fissure, and posteriorly by the central sulcus. Moving anterior to posterior, the inferior boundary was the sylvian fissure, then the white matter between the central sulcus and the inferior extent of the corpus callosum. This parcellation included BA 4, 6, and 44. The cingulate cortex anterior and superior boundaries were defined by the cingulate sulcus, inferiorly by the callosal sulcus and corpus callosum, and medially by the interhemispheric fissure. Moving anterior to posterior, the lateral boundary was initially a straight line across the white matter, connecting the depths of the cingulate and callosal sulci, then a straight line connecting the splenial sulcus to the tip of the lateral ventricle, and finally the splenial sulcus. This parcellation included BA 23-25, and 31.

The hippocampus was defined with the horn of the lateral ventricle as the dorsal and lateral boundary and the white matter separating the hippocampus from the entorhinal cortex as the ventral border (Rosene and Hoesen, 1987). The hippocampus was further divided into an anterior and a posterior portion, with the boundary delineated after the last coronal slice to include the uncus (Willard et al., 2011). The amygdala was defined following additional anatomical landmarks published for the macaque (Price et al., 1987) with the hippocampus as the posterior boundary, the beginning of the periamygdaloid cortex as the anterior boundary, the CSF as ventral border, white matter as ventrolateral boundary, and when this was not available due to low contrast, the rhinal fissure defined the ventromedial border. Intra and inter-rater reliability of volumes were assessed using intraclass correlation coefficients (ICCs). Using a subset of five subjects, two raters (K.M.R, C.F) manually adjusted the gray matter, white matter, and hippocampus segmentations with the following inter-rater reliability ICCs: gray matter $(r=.99)$, white matter $(r=.99)$, ICV $(r=.99)$, and hippocampus ( $\mathrm{r}=.67$ ). Intra-rater reliability using the same subset of five subjects was assessed for both raters with the following ICCs: gray matter $(r=.99 ; r=.93)$, white matter $(r=.99 ; r=.99)$, ICV $(r=.99$; $r=.99)$, hippocampus $(r=.74 ; r=.71)$, and amygdala $(r=.55)$.

\subsection{Statistical analysis}

Total brain volume, or intracranial volume (ICV), was calculated for each individual at both treatment conditions by adding total gray matter, white matter, and CSF volumes. In order to control for potential group differences in total ICV, all ROI volumes (cortical and subcortical) were adjusted for ICV volumes (e.g. gray matter/ICV). ICVadjusted ROI volumes were analyzed using repeated measures analysis of variance (rmANOVA) to test for between subject effects of social status (DOM; SUB), and the within subject effects of E2 treatment (E2; Control) and hemisphere (right; left). Additionally, for each cortical ROI (PFC, cingulate, frontal cortex) gray matter/ white matter ratios (GM/WM) were also calculated and analyzed using rmANOVA due to their relevance in understanding cortical neuropathological changes 
Table 1

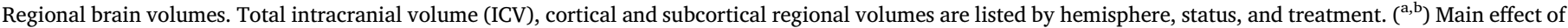

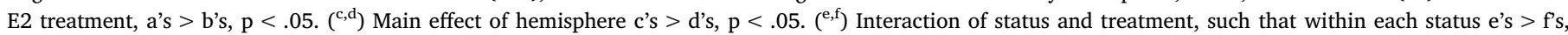

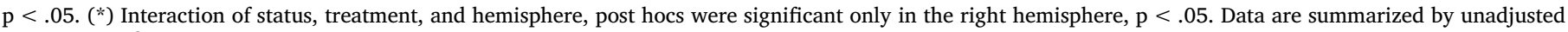
volumes $\left(\mathrm{mm}^{3} \pm\right.$ S.E. $)$.

\begin{tabular}{|c|c|c|c|c|c|}
\hline \multirow[t]{2}{*}{ Region } & \multirow[t]{2}{*}{ Hemisphere } & \multicolumn{2}{|c|}{ Dominant } & \multicolumn{2}{|c|}{ Subordinate } \\
\hline & & Control & Estradiol & Control & Estradiol \\
\hline \multicolumn{6}{|l|}{ Total } \\
\hline ICV & NA & $89449.7 \pm 2062.3$ & $88987.8 \pm 2030.6$ & $88641.1 \pm 1844.6$ & $88595.9 \pm 1816.3$ \\
\hline \multicolumn{6}{|l|}{ Prefrontal } \\
\hline \multirow[t]{2}{*}{ Gray Matter } & $\mathrm{R}$ & $2463.4 \pm 87.4^{d}$ & $2501.5 \pm 98.2^{d}$ & $2557.6 \pm 78.2^{d}$ & $2539.7 \pm 87.9^{d}$ \\
\hline & $\mathrm{L}$ & $2539.8 \pm 94.8^{c}$ & $2555.8 \pm 100.4^{\mathrm{c}}$ & $2604.9 \pm 84.8^{c}$ & $2604.4 \pm 89.8^{c}$ \\
\hline \multirow[t]{2}{*}{ White Matter } & $\mathrm{R}$ & $1076.5 \pm 37.6$ & $1101.3 \pm 38.1$ & $1105.7 \pm 33.7$ & $1110.3 \pm 34.0$ \\
\hline & $\mathrm{L}$ & $1088.4 \pm 38.6$ & $1093.6 \pm 41.1$ & $1079.1 \pm 34.6$ & $1075.5 \pm 36.8$ \\
\hline \multicolumn{6}{|l|}{ Frontal } \\
\hline \multirow[t]{2}{*}{ Gray Matter } & $\mathrm{R}$ & $2980.3 \pm 64.1^{\mathrm{a}, \mathrm{c}}$ & $2914.4 \pm 70.7^{b, c}$ & $2902.1 \pm 57.3^{\mathrm{a}, \mathrm{c}}$ & $2838.4 \pm 63.2^{b, c}$ \\
\hline & $\mathrm{L}$ & $2959.3 \pm 72.1^{\mathrm{a}, \mathrm{d}}$ & $2868.9 \pm 74.2^{\mathrm{b}, \mathrm{d}}$ & $2821.8 \pm 64.5^{\mathrm{a}, \mathrm{d}}$ & $2758.0 \pm 66.4^{b, d}$ \\
\hline \multirow[t]{2}{*}{ White Matter } & $\mathrm{R}$ & $1645.3 \pm 43.7$ & $1628.6 \pm 53.8$ & $1617.5 \pm 39.1$ & $1626.5 \pm 48.2$ \\
\hline & $\mathrm{L}$ & $1612.9 \pm 54.1$ & $1561.4 \pm 40.0$ & $1599.3 \pm 48.4$ & $1592.5 \pm 35.8$ \\
\hline \multicolumn{6}{|l|}{ Cingulate } \\
\hline \multirow[t]{2}{*}{ Gray Matter } & $\mathrm{R}$ & $872.6 \pm 27.3^{c, f, *}$ & $905.2 \pm 24.4^{\mathrm{c}, \mathrm{e}, *}$ & $903.3 \pm 24.5^{\mathrm{c}, \mathrm{e}, *}$ & $868 \pm 21.8^{\mathrm{c}, \mathrm{f}, *}$ \\
\hline & $\mathrm{L}$ & $843.9 \pm 30.2^{\mathrm{d}, \mathrm{f}}$ & $838.5 \pm 32.6^{\mathrm{d}, \mathrm{e}}$ & $842.9 \pm 27^{\mathrm{d}, \mathrm{e}}$ & $828.1 \pm 29.2^{\mathrm{d}, \mathrm{f}}$ \\
\hline \multirow[t]{2}{*}{ White Matter } & $\mathrm{R}$ & $220.9 \pm 8.4$ & $231.0 \pm 11.7$ & $230.3 \pm 7.5$ & $220.3 \pm 10.4$ \\
\hline & $\mathrm{L}$ & $233.0 \pm 8.4$ & $239.2 \pm 9.7$ & $225.0 \pm 7.5$ & $228.9 \pm 8.7$ \\
\hline \multicolumn{6}{|l|}{ Hippocampus } \\
\hline \multirow[t]{2}{*}{ All } & $\mathrm{R}$ & $433.0 \pm 10.8$ & $439.1 \pm 10.1$ & $455.2 \pm 9.6$ & $445.3 \pm 9.0$ \\
\hline & $\mathrm{L}$ & $435.2 \pm 13.2$ & $433.2 \pm 12.9$ & $463.1 \pm 11.8$ & $447.9 \pm 11.5$ \\
\hline \multirow[t]{2}{*}{ Posterior } & $\mathrm{R}$ & $222.0 \pm 8.2$ & $217.4 \pm 7.4$ & $230.6 \pm 7.3$ & $225.3 \pm 6.7$ \\
\hline & $\mathrm{L}$ & $231.7 \pm 8.4$ & $215.8 \pm 7.3$ & $231.1 \pm 7.5$ & $223.8 \pm 6.5$ \\
\hline \multirow[t]{2}{*}{ Anterior } & $\mathrm{R}$ & $210.9 \pm 8.6$ & $221.7 \pm 8.3$ & $224.6 \pm 7.7$ & $220.0 \pm 7.5$ \\
\hline & $\mathrm{L}$ & $203.5 \pm 9.7$ & $217.3 \pm 11.2$ & $232.0 \pm 8.7$ & $224.1 \pm 10$ \\
\hline \multicolumn{6}{|l|}{ Amygdala } \\
\hline \multirow[t]{2}{*}{ All } & $\mathrm{R}$ & $323.7 \pm 10.1^{d}$ & $331.2 \pm 12.5^{\mathrm{d}}$ & $309.6 \pm 9.1^{d}$ & $302.9 \pm 11.2^{\mathrm{d}}$ \\
\hline & $\mathrm{L}$ & $345.9 \pm 10.5^{c}$ & $352.9 \pm 11.8^{\mathrm{c}}$ & $321.8 \pm 9.4^{c}$ & $331.8 \pm 10.6^{\mathrm{c}}$ \\
\hline
\end{tabular}

(e.g. during aging). Finally, raw volume data (ICV unadjusted) were analyzed using the same rmANOVA models. Results are summarized as unadjusted ICV, gray matter, white matter, and subcortical volumes $\left(\mathrm{mm}^{3} \pm\right.$ S.E. $)$.

\section{Results}

\subsection{Total brain volume}

There was no significant effect of status $\left(\mathrm{F}_{1,16}=.05, \mathrm{p}=.83\right.$, $\left.\eta_{\mathrm{p}}^{2}<.01\right)$, E2 treatment $\left(\mathrm{F}_{1,16}=.42, \mathrm{p}=.52, \eta_{\mathrm{p}}^{2}=.03\right)$, or a status by $\mathrm{E} 2$ treatment interaction $\left(\mathrm{F}_{1,16}=.29, \mathrm{p}=.60, \eta_{\mathrm{p}}^{2}=.02\right)$, on total brain volume (Table 1).

\subsection{Lobar volumes}

\subsubsection{Gray matter volume}

Results and raw data are listed in Table 1 . There was a main effect of treatment on gray matter volume in the frontal cortex $\left(\mathrm{F}_{1,16}=11.19, \mathrm{p}\right.$ $=.004, \eta_{\mathrm{p}}^{2}=.41$, Fig. 2A). Specifically, E2 replacement reduced frontal gray matter volume compared to the control condition (no E2), but there were no effects of status $\left(\mathrm{F}_{1,16}=1.77, \mathrm{p}=.20, \eta_{\mathrm{p}}^{2}=.10\right)$ or status by treatment interaction effect $\left(\mathrm{F}_{1,16}<.001, \mathrm{p}>.99, \eta_{\mathrm{p}}^{2}<.01\right)$. There was a significant status by treatment by hemisphere interaction $\left(\mathrm{F}_{1,16}=5.24, \mathrm{p}=.036, \eta_{\mathrm{p}}^{2}=.25\right)$ on cingulate cortex gray matter volume, but no main effects of status $\left(\mathrm{F}_{1,16}<.001, \mathrm{p}=.97, \eta_{\mathrm{p}}^{2}<.001\right)$ or treatment $\left(\mathrm{F}_{1,16}=.27, \mathrm{p}=.61, \eta_{\mathrm{p}}^{2}=.02\right)$ were found. Supporting this three-way interaction in cingulate gray matter volume, there was also a main effect of hemisphere $\left(\mathrm{F}_{1,16}=9.35, \mathrm{p}=.008, \eta_{\mathrm{p}}^{2}=.37\right)$ and an interaction effect of status by treatment $\left(F_{1,16}=8.65, p=.010, \eta_{p}^{2}\right.$ $=.35$ ). As shown in Fig. 2 (B) cingulate gray matter volumes in the right hemisphere were differentially affected by status and treatment, as DOMs had greater gray matter volume in the E2 condition compared to control treatment $(\mathrm{p}=.012)$ and SUBs had decreased gray matter volume during the E2 condition compared to the control treatment ( $\mathrm{p}$ $=.010$ ). Post hoc tests showed no significant status difference between
A

E2 Decreased Bilateral Frontal Cortex Gray Matter

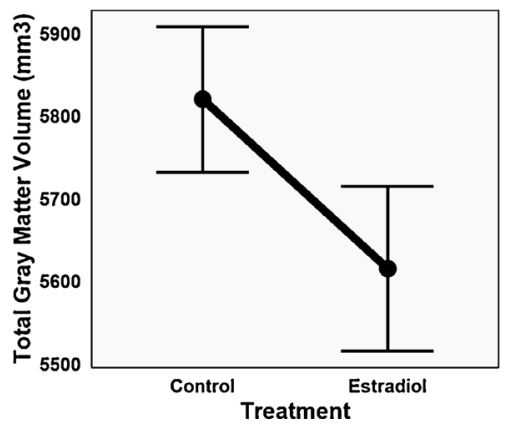

B Status Modified E2 Effects on Right Cingulate Cortex Gray Matter

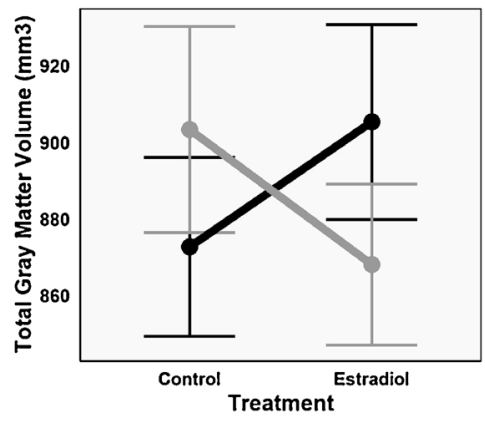

Fig. 2. (A) Main effects of E2 treatment on frontal gray matter volume $(\mathrm{p}=.004)$, E2 decreased gray matter volume independent of status. (B) Status by Treatment by Hemisphere effect on cingulate gray matter volume ( $\mathrm{p}=$ .036), post-hoc analyses showed DOMs increased gray matter volume between the control and E2 conditions ( $\mathrm{p}=.012)$ and SUBs decreased gray matter volume between the control and E2 conditions ( $\mathrm{p}=.010)$. Data are reported as unadjusted mean volume changes $\left(\mathrm{mm}^{3}\right) \pm$ SEM. 
Table 2

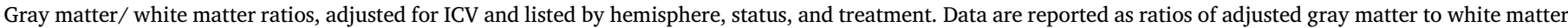

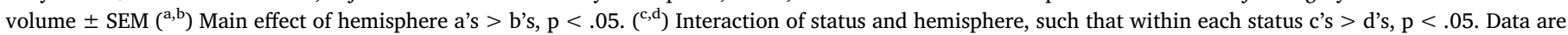
summarized by unadjusted volumes $\left(\mathrm{mm}^{3} \pm\right.$ S.E. $)$.

\begin{tabular}{|c|c|c|c|c|c|}
\hline \multirow[t]{2}{*}{ Region } & \multirow[t]{2}{*}{ Hemisphere } & \multicolumn{2}{|c|}{ Dominant } & \multicolumn{2}{|c|}{ Subordinate } \\
\hline & & Control & Estradiol & Control & Estradiol \\
\hline \multicolumn{6}{|c|}{ Prefrontal } \\
\hline & $\mathrm{R}$ & $2.30 \pm 0.07^{\mathrm{b}, \mathrm{d}}$ & $2.28 \pm 0.07^{\mathrm{b}, \mathrm{d}}$ & $2.32 \pm 0.06^{\mathrm{b}, \mathrm{d}}$ & $2.29 \pm 0.07^{\mathrm{b}, \mathrm{d}}$ \\
\hline & $\mathrm{L}$ & $2.35 \pm 0.06^{\mathrm{a}, \mathrm{c}}$ & $2.35 \pm 0.07^{\mathrm{a}, \mathrm{c}}$ & $2.41 \pm 0.06^{\mathrm{a}, \mathrm{c}}$ & $2.42 \pm 0.06^{\mathrm{a}, \mathrm{c}}$ \\
\hline \multicolumn{6}{|l|}{ Frontal } \\
\hline & $\mathrm{R}$ & $1.82 \pm 0.04$ & $1.80 \pm 0.07$ & $1.8 \pm 0.04$ & $1.75 \pm 0.06$ \\
\hline & $\mathrm{L}$ & $1.84 \pm 0.05$ & $1.84 \pm 0.05$ & $1.77 \pm 0.04$ & $1.74 \pm 0.05$ \\
\hline \multicolumn{6}{|c|}{ Cingulate } \\
\hline & $\mathrm{R}$ & $3.96 \pm 0.13^{a}$ & $3.97 \pm 0.16^{\mathrm{a}}$ & $3.95 \pm 0.11^{\mathrm{a}}$ & $3.98 \pm 0.14^{\mathrm{a}}$ \\
\hline & $\mathrm{L}$ & $3.64 \pm 0.12^{b}$ & $3.55 \pm 0.14^{b}$ & $3.76 \pm 0.11^{b}$ & $3.62 \pm 0.13^{b}$ \\
\hline
\end{tabular}

right hemisphere volumes during the $\mathrm{E} 2$ treatment $(\mathrm{p}=.26)$ or the no treatment condition $(\mathrm{p}=.28)$. In addition, there were significant effects of hemisphere detected on both frontal gray matter $\left(\mathrm{F}_{1,16}=6.94\right.$, $\left.\mathrm{p}=.018, \eta_{\mathrm{p}}^{2}=.30\right)$ and PFC gray matter volumes $\left(\mathrm{F}_{1,16}=9.07, \mathrm{p}=\right.$ $.008, \eta_{\mathrm{p}}^{2}=.36$ ). Frontal gray matter volumes were larger in the right hemisphere while PFC gray matter volumes were larger in the left hemisphere. Similar results were seen for data unadjusted for ICVs.

\subsubsection{White matter volumes}

Results and raw data are listed in Table 1 . There were no main or interaction effects of status, E2, or hemisphere on PFC, frontal, or cingulate white matter volumes.

\subsubsection{Gray Matter/White matter ratio}

Results and raw data are listed in Table 2. There were significant hemispheric lateralization effects in GM/WM ratios in the PFC $\left(\mathrm{F}_{1,16}=\right.$ 62.71, $\left.\mathrm{p}<.001, \eta_{\mathrm{p}}^{2}=.80\right)$ and cingulate cortex $\left(\mathrm{F}_{1,16}=12.82, \mathrm{p}=\right.$ $.002, \eta_{\mathrm{p}}^{2}=.45$ ). The PFC showed an increased GM/WM ratio (gray matter $>$ white matter) in the left compared with the right hemisphere (Right $=2.30 \pm .05$; Left $=2.38 \pm .04$ ) while the cingulate cortex showed the reverse pattern, with an increased GM/WM ratio in the right compared with the left hemisphere (Right $=3.97 \pm .08$; Left $=$ $3.64 \pm .07)$. Furthermore, there was an interaction effect between status and hemisphere in the PFC $\left(\mathrm{F}_{1,16}=7.81, \mathrm{p}=.013, \eta_{\mathrm{p}}^{2}=.33\right)$. Post hoc analysis, however, did not detect any significant interactions. $\mathrm{GM} / \mathrm{WM}$ ratios were bigger in the left than in the right hemisphere in both DOMs ( $\mathrm{p}=.003$; Right $=2.29 \pm .07$; Left $=2.35 \pm .07)$ and SUBs $(\mathrm{p}<.001$; Right $=2.30 \pm .06$; Left $=2.42 \pm .06$ ), and there were no GM/WM ratio differences between SUBs and DOMs in either the left hemisphere $(\mathrm{p}=.43)$ or right hemisphere $(\mathrm{p}=.92)$. Similar results were seen for data unadjusted for ICVs.

\subsection{Hippocampus}

No main or interaction effects were observed in the hippocampus. There was no effect of status $\left(\mathrm{F}_{1,16}=2.51, \mathrm{p}=.13, \eta_{\mathrm{p}}^{2}=.14\right)$, treatment $\left(\mathrm{F}_{1,16}=.17, \mathrm{p}=.69, \eta_{\mathrm{p}}^{2}=.01\right)$, or a status by treatment interaction $\left(\mathrm{F}_{1,16}=.79, \mathrm{p}=.39, \eta_{\mathrm{p}}^{2}=.05\right)$ on total hippocampal volumes. Furthermore, when divided into anterior and posterior hippocampus, no effects of status (anterior: $\mathrm{F}_{1,16}=1.23, \mathrm{p}=.28, \eta_{\mathrm{p}}^{2}=.07$; posterior: $\mathrm{F}_{1,16}=.84, \mathrm{p}=.37, \eta_{\mathrm{p}}^{2}=.05$ ), treatment (anterior: $\mathrm{F}_{1,16}=.48, \mathrm{p}=$ $.50, \eta_{\mathrm{p}}^{2}=.03$; posterior: $\mathrm{F}_{1,16}=2.44, \mathrm{p}=.14, \eta_{\mathrm{p}}^{2}=.13$ ), or status by treatment effects were detected on these volumes (anterior: $\mathrm{F}_{1,16}=$ $3.20, \mathrm{p}=.09, \eta_{\mathrm{p}}^{2}=.17$; posterior: $\left.\mathrm{F}_{1,16}=.07, \mathrm{p}=.79, \eta_{\mathrm{p}}^{2}=.01\right)$. There were no effects of hemisphere (total: $\mathrm{F}_{1,16}=.34, \mathrm{p}=.57, \eta_{\mathrm{p}}^{2}=$ .02 ; anterior: $\mathrm{F}_{1,16}=.02, \mathrm{p}=.89, \eta_{\mathrm{p}}^{2}<.01$; posterior: $\mathrm{F}_{1,16}=.36, \mathrm{p}=$ $.58, \eta_{\mathrm{p}}^{2}=.02$ ), or status by hemisphere (total: $\mathrm{F}_{1,16}=1.13, \mathrm{p}=.30, \eta_{\mathrm{p}}^{2}$ $=.07$; anterior: $\mathrm{F}_{1,16}=3.51, \mathrm{p}=.08, \eta_{\mathrm{p}}^{2}=.18$; posterior: $\mathrm{F}_{1,16}=.36$, $\mathrm{p}=.56, \eta_{\mathrm{p}}^{2}=.02$ ), treatment by hemisphere (total: $\mathrm{F}_{1,16}=1.17, \mathrm{p}=$
$.30, \eta_{\mathrm{p}}^{2}=.07$; anterior: $\mathrm{F}_{1,16}=.01, \mathrm{p}=.91, \eta_{\mathrm{p}}^{2}<.01$; posterior: $\mathrm{F}_{1,16}$ $=2.07, \mathrm{p}=.17, \eta_{\mathrm{p}}^{2}=.12$ ), or status by treatment by hemisphere interaction effects (total: $\mathrm{F}_{1,16}=.04, \mathrm{p}=.84, \eta_{\mathrm{p}}^{2}<.01$; anterior: $\mathrm{F}_{1,16}$ $=.57, \mathrm{p}=.46, \eta_{\mathrm{p}}^{2}=.03$; posterior: $\mathrm{F}_{1,16}=.91, \mathrm{p}=.36, \eta_{\mathrm{p}}^{2}=.05$ ). Similar results were seen for data unadjusted for ICVs. Results and raw data are listed in Table 1.

\subsection{Amygdala}

There was a main effect of hemisphere $\left(\mathrm{F}_{1,16}=30.53, \mathrm{p}<.001, \eta_{\mathrm{p}}^{2}\right.$ $=.66$ ), such that left amygdala volumes were larger than the right. There were no main effects of status $\left(\mathrm{F}_{1,16}=2.54, \mathrm{p}=.13, \eta_{\mathrm{p}}^{2}=.14\right)$ or treatment $\left(\mathrm{F}_{1,16}=1,28, \mathrm{p}=.27, \eta_{\mathrm{p}}^{2}=.07\right)$ and no interaction effects including status by treatment $\left(\mathrm{F}_{1,16}=.58, \mathrm{p}=.46, \eta_{\mathrm{p}}^{2}=.04\right)$, status by hemisphere $\left(\mathrm{F}_{1,16}=.03, \mathrm{p}=.87, \eta_{\mathrm{p}}^{2}<.01\right)$, treatment by hemisphere $\left(\mathrm{F}_{1,16}=1.54, \mathrm{p}=.23, \eta_{\mathrm{p}}^{2}=.09\right)$, or status by treatment by hemisphere $\left.\left(\mathrm{F}_{1,16}=1.58, \mathrm{p}=.23, \eta_{\mathrm{p}}^{2}=.09\right)\right)$. Similar results were seen for data unadjusted for ICVs. Results and raw data are listed in Table 1.

\section{Discussion}

Our findings demonstrated that both E2 and subordinate social status were associated with region-specific structural changes in the adult female brain. In particular, one month of E2 treatment in ovariectomized adult female macaques caused significant decreases in frontal cortex gray matter volume. Furthermore, E2 altered structure in the right cingulate cortex, depending on social status, increasing gray matter volume in DOMs, but decreasing it in SUBs. These data suggest that a background of chronic social stress interacts with E2 to alter brain structure.

We hypothesized that subordinate social status would be associated with reduced hippocampal, PFC, frontal and cingulate volumes and increased amygdala volumes, however, our data showed no such effects on any regional or total brain volumes, independent of E2 treatment. These findings are contradictory to the nonhuman primate (Sallet et al., 2011; Sanchez et al., 1998; Spinelli et al., 2009) and rodent stress literature described in the introduction. There are at least three possible factors that may have resulted in our negative findings. First, although Sallet et al., reported reduced gray matter density in the rostral PFC in subordinate compared to higher ranking young adult males (Sallet et al., 2011), social subordination is a different experience of chronic stress for macaque males and females (Wilson, 2016). Thus, the consequences of subordination on brain structure are likely sex dependent and may be modified by sex steroid hormones, as demonstrated by the effects of chronic stress in rodents as well as in our findings. Second, Sallet et al., measured structural changes using deformation-based morphometry (DBM), a method more sensitive to small changes in gray matter volume compared to our methods, which measured larger 
regional volumes. It may be that the structural brain changes associated with subordinate social status in female rhesus monkeys are smaller in area than our methods allowed us to detect. Third, the majority of gross regional alterations in rhesus monkey brain volumes associated with stress have been reported mainly in studies of stress exposure during development, when brain organization is still underway, including prenatal and early-life exposure (Godfrey et al., 2013; Sanchez et al., 1998; Spinelli et al., 2009). In these studies, chronic stress or GCs were associated with decreased corpus callosum in males (Sanchez et al., 1998), decreased frontal and cingulate cortical volumes in both males and females (Spinelli et al., 2009), and increased amygdala volumes in juvenile females (Godfrey et al., 2013). In the current study, our goal was to examine the specific structural effects of subordinate social status imposed during adulthood, actually controlling for exposure to early life stress by using adult females that were born and raised into middle ranking matrilines within their natal groups (Jarrell et al., 2008). Thus, we suspect that subordinate social status may have larger regional effects when experienced during development and into adulthood and suggest that future analyses examining the impact of chronic stress during adulthood use methods that allow for detection of small local changes in brain volumes.

A critical finding in our data was the modifying effect of subordinate social status on E2's impact on the right cingulate cortex, wherein chronic social stress and E2 reduced cortical gray matter volume. However, these findings were contrary to our hypothesis that physiological relevant levels of E2 treatment in the context of subordinate social status (high stress) would result in increased cortical gray matter in the PFC, frontal, and cingulate cortices. Our original hypothesis was based primarily on histology studies of neuronal morphology in rodents (Garrett and Wellman, 2009; Shansky et al., 2010), while our methods examined averaged changes in gray matter volumes across large lobar parcellations. Therefore, it is possible that the granular changes observed in rodent studies do no readily scale to changes observable in structural MRI scans. However, our results demonstrated that E2 and chronic social stress can have deleterious effects on the broader cingulate cortex. Findings from our lab in this same model of social subordination demonstrated social status dependent alterations in cingulate (subgenual) resting-state functional connectivity (FC) with the amygdala following E2 treatment, suggesting that the structural changes identified may have functional correlates (Reding et al., 2019). Right amygdala-subgenual FC was greater in DOMs than SUBs during the control (no treatment) condition, and E2 treatment resulted in increased SUB FC and decreased DOM FC. Thus, both the structural and functional data showed an interaction with status and E2 in the cingulate cortex, suggesting high sensitivity of the underlying neurobiology to their combined effects in this region.

Although the implication of our findings for behavior, and ultimately stress-related phenotypes in women, are beyond the scope of our study, our data do provide some evidence that the cingulate cortex may have a specific vulnerability to chronic stress in females (or may be a substrate that is involved in an adaptation to chronic stress) that subsequently alters E2s effects to produce changes in brain function and resultant behavior. In humans, reductions in cingulate cortical volume have been linked with post-traumatic stress disorder (Karl et al., 2006), and reductions in subgenual cingulate volume are characteristic alterations in individuals with major depressive disorder (Drevets et al., 2008), although they are not always detected (Hamani et al., 2011). Data on normally cycling women without psychopathology have also demonstrated negative correlation between circulating E2 and anterior cingulate volumes across the menstrual cycle (Catenaccio et al., 2016). It is possible that our finding of E2-induced changes in cingulate volumes dependent on chronic stress in female rhesus monkey can explain some of the disparities in the human literature regarding the cingulate volume effects seen in clinical populations with stress-related phenotypes.

The data from the present study demonstrated that approximately one month of treatment with a physiologically relevant dose of E2 both reduced frontal gray matter volume and interacted with social subordination to reduce cingulate gray matter volume in SUBs and increase it in DOMs. We had hypothesized, based on the rodent literature, that E2 treatment would increase cortical gray matter in the absence of stress and chronic stress could further potentiate these effects. However, our findings of decreased gray matter volumes may be related to $\mathrm{E} 2$ treatment duration, which did not mimic paradigms used in rodent studies or naturalistic changes across the menstrual cycle (but was comparable to short-term estrogen replacement therapy [ERT]). The effects of chronic versus acute E2 treatment on neuronal morphology have not been systematically compared, particularly across species. For example, in the hippocampus, previous studies have demonstrated that E2's protective effect on spine density were seen $24 \mathrm{~h}$ following acute E2 treatment and persisted for up to 9 days (Gould et al., 1990). Likewise, chronic E2 administration (approximately 5 weeks) resulted in increased hippocampal dendritic arborization and spine density (McLaughlin et al., 2010). Less is known about the effect of chronic or acute E2 treatment in rodent PFC and amygdala dendritic morphology, however, the data presented in the introduction varied from one week (Shansky et al., 2010) to approximately two weeks (Garrett and Wellman, 2009), which also exceeded the typical rodent estrus cycle of four to five days. In the literature on post-menopausal women, the effects of ERT are thought to be moderated by age and/or timing of treatment following menopause, among other factors, wherein the beneficial effects of ERT on brain structure and cognition are only seen if treatment is initiated very soon after menopause (Wnuk et al., 2012). Delays in ERT after menopause enhanced structural atrophy in the prefrontal cortex and increased cognitive impairment (Resnick et al., 2009). These disparate effects of ERT that are related to treatment timing may be because estrogen's protective effects can quickly turn to apoptotic in stressed or damaged cells (Brinton, 2008). In general, E2's effects on neural morphology and brain structure in adulthood, and the effect of treatment duration, appear to function in a region-specific manner and more data are needed to determine its effects within specific cortical regions.

Due to the literature on the impact of delayed ERT in postmenopausal women, we must consider the possibility that chronic stress, aging, or the lack of estrogen itself for a critical period of time, can all damage neuronal integrity, and thus, the reintroduction of E2 to compromised neurons may change their effects from beneficial to damaging. However, we do not think this is the case in our study and believe that our finding of differential impact of $\mathrm{E} 2$ on the cingulate cortex of DOM and SUB females is most likely due to the impact of chronic stress, and not of age or timing of E2 treatment following ovariectomy. There are several reasons for our interpretation. First, in the current study, all females were ovariectomized for $\sim 6$ years and were approximately 14 years of age at the time of their scans, comparable to middle age premenopausal women. In other words, there were no social status differences in age at ovariectomy or time from ovariectomy. Second, and importantly, since ovariectomy, the subjects had intermittent exposure to E2 lasting approximately 2-4 weeks and/ or progesterone for similar durations (Michopoulos et al., 2009, 2011; Reding et al., 2012). In these previous studies, there were robust effects of E2 treatment on outcome measures including sociosexual behavior (Reding et al., 2012), hypothalamic pituitary gonadal (HPG) axis negative feedback (Michopoulos et al., 2009), and serotonergic function (Michopoulos et al., 2011). Thus, although it is likely that there are differences in exposure to estrogens between gonadally intact females and the ovariectomized females in our sample, we believe that our experimental design allowed us to address the impact of chronic stress on the effects of E2 (independent of the ovarian cycle and thus additional effects of progesterone) on brain structure after a short treatment period (4-6 weeks). Therefore, we believe that the current findings suggest that the effects of E2 on cingulate cortex are social status-dependent and due to E2's negative effects (e.g. reduced synaptic density 
and dendritic arborization/length) in chronically stressed SUB females, in contrast to the trophic effects of E2 in non-stressed DOM females.

Hemispheric lateralization effects (main and interaction) were also detected in several regions. Studies on primate brain lateralization suggest that humans have greater frontal gray matter volumes in the left hemisphere, and comparative phylogeny of monkey species show the reverse pattern (Smaers et al., 2011), and this same trend is seen in rhesus monkeys (Falk et al., 1990). The functional implications of lateralized effects are still unclear and a source of debate in the literature.

Two limitations of our studies are the lack of information about the cellular mechanisms that account for volumetric differences (i.e., are they reflecting changes in dendritic length and/or branching, or neuronal or glial density or size?) as well as an understanding of the biological signals that mediate the effects of chronic stress and E2 treatment. To address the first limitation, rodent studies comparing reductions in cingulate and hippocampal volumes obtained using sMRI with postmortem histological analyses from the same animals suggest that volume loss is associated with reduced density of synaptic spines, dendritic length, but not total number or surface area of neurons or glia (Kassem et al., 2013). However, post-mortem data from individuals diagnosed with major depression suggest that reduced subgenual cingulate volumes are due to a reduction of the number of glia (Ongur et al., 1998), while volume reductions of PFC regions are associated with reduced cell volumes of both neurons and glia (Rajkowska, 2000). Further studies are needed to address the underlying cellular and molecular mechanisms of the volumetric changes reported in our study and to understand the neurobiological mechanisms underlying stressrelated changes in adult female monkey brain structure. Taken together, our data suggest that particular attention needs to be paid to hormonal state in future neuroimaging analyses on brain regional volumes.

In regard to the second limitation, there are several potential mechanisms by which stress and E2 may alter brain structure. Stress hormones, including cortisol, and E2 can act on GC receptors and estrogen receptors, which are expressed throughout the neocortex, including the PFC, frontal and cingulate cortices (Diorio et al., 1993; Morimoto et al., 1996; Pau et al., 1998; Sánchez et al., 2000; Shughrue et al., 1997). As described above, we suspect that subordinate social status altered brain structure in a way that modified the impact of E2 to result in E2-induced decreases in cingulate gray matter volume in chronically stressed females. However, the reverse may be true, that E2 altered the effects of chronic stress on gray matter volume. Unfortunately, our current study design did not allow us to test either of these two hypotheses, as we did not collect cortisol data to study the interactions between gonadal and stress hormones to help us explain the structural brain effects reported. Previous studies from our lab have shown that SUB females exhibit stress-related phenotypes that include alterations in the HPA axis, including impaired GC negative feedback and flattened circadian cortisol secretion in ovariectomized SUBs without E2 replacement (Michopoulos et al., 2012b). In addition, we have shown that replacing E2 in a dose-dependent manner, using a series of increasing E2-replacement regimens, restores basal cortisol levels in SUBs while having no effect on DOMs (Toufexis et al., 2014). However, replacing E2 also increased the cortisol response $30 \mathrm{~min}$ following an acute stressor only in SUBs. These previous findings suggest that subordinate social status (i.e., SUB) is associated with impaired HPA axis function while the addition of E2 can result in a hyperresponsive HPA axis (Toufexis et al., 2014). Although we did not measure HPA axis function in the current study, our previous reports suggest that the observed decrease in cingulate cortex volume in SUBs is likely concurrent with an E2 mediated hyper-responsive HPA axis. Thus, it is possible that a hyper-responsive HPA axis may have mediated the E2-induced reductions in cingulate cortex structural volume observed in SUBs in the current study, explaining the opposite E2 trophic effects on DOMs cingulate volume.

In conclusion, social subordination and E2 treatment in adult female rhesus monkeys both induced changes in cortical gray matter volumes. This study presents novel data supporting the plasticity of the adult female primate brain in response to social experience and changing hormonal environments. Our data suggest that exposure to chronic stress imposed during adulthood by subordinate social status alters subsequent structural effects of E2 on specific cortical regions. Importantly, social status effects on brain volumes are not detected without concomitant treatment with $\mathrm{E} 2$, suggesting that subordinate social status has profound, yet dichotomous, effects on E2. Importantly, the E2- and status-related structural changes reported here are striking, as they are visible following only one month of E2 treatment in ovariectomized females and are found despite periods of time without endogenous ovarian hormones. Together these results underscore the plasticity of the female primate brain and shed light on effects of subordinate social status and E2 treatment on it, providing insights into possible mechanism of adaptation to the social and hormonal environments that may exacerbate risk of developing stress-related phenotypes and disorders in women.

\section{Funding}

This work was supported by grants from the National Institutes of Health including R01 MH081816, R01 MH081816-04S1, R01 MH078105-S1, R01 MH091645, U54 EB005149, U54 HD079124, and P51 OD11132 (Yerkes National Primate Research Center [YNPRC] Base grant). The YNPRC is fully accredited by the American for the Assessment and Accreditation of Laboratory Care (AAALAC), International.

\section{Declaration of Competing Interest}

None.

\section{Acknowledgements}

The study was conducted with the technical expertise of Jennifer Whitley, Natalie Brutto, Shannon Moss, Dr. Jodi Godfrey, Jonathon Lowe, and Erin O'Sheill. We would also like to acknowledge the assistance of Carline $\mathrm{Fu}$ in processing neuroimaging data, including quality control and brain segmentation.

\section{References}

Asher, J., Michopoulos, V., Reding, K.M., Wilson, M.E., Toufexis, D., 2013. Social stress and the polymorphic region of the serotonin reuptake transporter gene modify oestradiol-induced changes on central monoamine concentrations in female rhesus monkeys. J. Neuroendocrinol. 25, 321-328.

Bernstein, I.S., Gordon, T.P., Rose, R.M., 1974. Aggression and social controls in rhesusmonkey (macaca-mulatta) groups revealed in group formation studies. Folia Primatol. 21, 81-107.

Brinton, R.D., 2008. The healthy cell bias of estrogen action: mitochondrial bioenergetics and neurological implications. Trends Neurosci. 31, 529-537.

Catenaccio, E., Mu, W., Lipton, M.L., 2016. Estrogen- and progesterone-mediated structural neuroplasticity in women: evidence from neuroimaging. Brain Struct. Funct. 221, 3845-3867.

Diorio, D., Viau, V., Meaney, M., 1993. The role of the medial prefrontal cortex (cingulate gyrus) in the regulation of hypothalamic-pituitary-adrenal responses to stress. J. Neurosci. 13, 3839-3847.

Drevets, W.C., Savitz, J., Trimble, M., 2008. The subgenual anterior cingulate cortex in mood disorders. CNS Spectr. 13, 663-681.

Falk, D., Hildebolt, C., Cheverud, J., Vannier, M., Criss Helmkamp, R., Konigsberg, L., 1990. Cortical asymmetries in frontal lobes of Rhesus monkeys ( $<\mathrm{i}>$ Macaca mulatta $</$ i $>$ ). Brain Res. 512, 40-45.

Garrett, J.E., Wellman, C.L., 2009. Chronic stress effects on dendritic morphology in medial prefrontal cortex: sex differences and estrogen dependence. Neuroscience 162, 195-207.

Godfrey, J.R., Howell, B.R., Mummert, A., Shi, Y., Styner, M., Sanchez, M.M., Wilson, M., 2013. Experimental Delay of Puberty Causes Structural Brain Alterations in Female Rhesus Macaques. Society for Neuroscience, San Diego.

Godfrey, J.R., Diaz, M.P., Pincus, M., Kovacs-Balint, Z., Feczko, E., Earl, E., MirandaDominguez, O., Fair, D., Sanchez, M.M., Wilson, M.E., Michopoulos, V., 2018. Diet matters: glucocorticoid-related neuroadaptations associated with calorie intake in 
female rhesus monkeys. Psychoneuroendocrinology 91, 169-178.

Gould, E., Woolley, C.S., Frankfurt, M., McEwen, B.S., 1990. Gonadal steroids regulate dendritic spine density in hippocampal pyramidal cells in adulthood. J. Neurosci. 10, 1286-1291.

Hamani, C., Mayberg, H., Stone, S., Laxton, A., Haber, S., Lozano, A.M., 2011. The subcallosal cingulate gyrus in the context of major depression. Biol. Psychiatry 69, 301-308.

Jarrell, H., Hoffman, J.B., Kaplan, J.R., Berga, S., Kinkead, B., Wilson, M.E., 2008. Polymorphisms in the serotonin reuptake transporter gene modify the consequences of social status on metabolic health in female rhesus monkeys. Physiol. Behav. 93 807-819.

Joels, M., Karst, H., Krugers, H.J., Lucassen, P.J., 2007. Chronic stress: implications for neuronal morphology, function and neurogenesis. Front. Neuroendocrinol. 28, 72-96.

Karl, A., Schaefer, M., Malta, L.S., Dorfel, D., Rohleder, N., Werner, A., 2006. A metaanalysis of structural brain abnormalities in PTSD. Neurosci. Biobehav. Rev. 30, 1004-1031.

Kassem, M.S., Lagopoulos, J., Stait-Gardner, T., Price, W.S., Chohan, T.W., Arnold, J.C., Hatton, S.N., Bennett, M.R., 2013. Stress-induced grey matter loss determined by mri is primarily due to loss of dendrites and their synapses. Mol. Neurobiol. 47, 645-661.

Kendler, K.S., Kessler, R.C., Neale, M.C., Heath, A.C., Eaves, L.J., 1993. The prediction of major depression in women: toward an integrated etiologic model. Am. J. Psychiatry 150, 1139-1148.

Kessler, R.C., 2003. Epidemiology of women and depression. J. Affect. Disord. 74, 5-13.

Knickmeyer, R.C., Styner, M., Short, S.J., Lubach, G.R., Kang, C., Hamer, R., Coe, C.L., Gilmore, J.H., 2010. Maturational trajectories of cortical brain development through the pubertal transition: unique species and sex differences in the monkey revealed through structural magnetic resonance imaging. Cereb. Cortex 20, 1053-1063.

McEwen, B.S., 1999. Stress and hippocampal plasticity. Annu. Rev. Neurosci. 22, $105-122$

McLaughlin, K.J., Wilson, J.O., Harman, J., Wright, R.L., Wieczorek, L., Gomez, J., Korol, D.L., Conrad, C.D., 2010. Chronic 17beta-estradiol or cholesterol prevents stress-induced hippocampal CA3 dendritic retraction in ovariectomized female rats: possible correspondence between CA1 spine properties and spatial acquisition. Hippocampus 20, 768-786.

Michopoulos, V., Berga, S.L., Kaplan, J.R., Wilson, M.E., 2009. Social subordination and polymorphisms in the gene encoding the serotonin transporter enhance estradiol inhibition of luteinizing hormone secretion in female rhesus monkeys. Biol. Reprod. 81, 1154-1163.

Michopoulos, V., Berga, S.L., Wilson, M.E., 2011. Estradiol and progesterone modify the effects of the serotonin reuptake transporter polymorphism on serotonergic responsivity to citalopram. Exp. Clin. Psychopharmacol. 19, 401-408.

Michopoulos, V., Higgins, M., Toufexis, D., Wilson, M.E., 2012a. Social subordination produces distinct stress-related phenotypes in female rhesus monkeys. Psychoneuroendocrinology 37, 1071-1085.

Michopoulos, V., Reding, K.M., Wilson, M.E., Toufexis, D., 2012b. Social subordination impairs hypothalamic-pituitary-adrenal function in female rhesus monkeys. Horm. Behav, 62, 389-399.

Michopoulos, V., Toufexis, D., Wilson, M.E., 2012c. Social stress interacts with diet history to promote emotional feeding in females. Psychoneuroendocrinology 37 , 1479-1490.

Morimoto, M., Morita, N., Ozawa, H., Yokoyama, K., Kawata, M., 1996. Distribution of glucocorticoid receptor immunoreactivity and mRNA in the rat brain: an immunohistochemical and in situ hybridization study. Neurosci. Res. 26, 235-269.

Ongur, D., An, X., Price, J.L., 1998. Prefrontal cortical projections to the hypothalamus in macaque monkeys. J. Comp. Neurol. 401, 480-505.

Pau, C.Y., Pau, K.Y., Spies, H.G., 1998. Putative estrogen receptor beta and alpha mRNA expression in male and female rhesus macaques. Mol. Cell. Endocrinol. 146, 59-68.

Paxinos, G., Huang, X.F., Toga, A.W., 2000. The Rhesus Monkey Brain in Stereotaxic Coordinates. Academic Press, San Diego, CA.

Price, J.L., Russcher, F.T., Amaral, D.G., 1987. The limbic region: II. The amygdaloid complex. In: Björklund, A., Hökfelt, T., Swanson, L.M. (Eds.), Handbook of Chemical Neuroanatomy. Elsevier; Sole distributors for the USA and Canada, Elsevier Science Pub. Co., Amsterdam; New York, NY.

Radley, J.J., Anderson, R.M., Hamilton, B.A., Alcock, J.A., Romig-Martin, S.A., 2013. Chronic stress-induced alterations of dendritic spine subtypes predict functional decrements in an hypothalamo-pituitary-adrenal-inhibitory prefrontal circuit. J. Neurosci. 33 14379-+.

Rajkowska, G., 2000. Postmortem studies in mood disorders indicate altered numbers of neurons and glial cells. Biol. Psychiatry 48, 766-777.

Reding, K.M., Grayson, D.S., Miranda-Dominguez, O., Ray, S., Wilson, M.E., Toufexis, D., Fair, D.A., Sanchez, M.M., 2019. Effects of social subordination and estradiol on resting-state prefrontal and amygdala functional connectivity in adult female rhesus monkeys. J. Neuroendocrinol (in press).

Reding, K., Michopoulos, V., Wallen, K., Sanchez, M., Wilson, M.E., Toufexis, D., 2012. Social status modifies estradiol activation of sociosexual behavior in female rhesus monkeys. Horm. Behav. 62, 612-620.

Resnick, S.M., Espeland, M.A., Jaramillo, S.A., Hirsch, C., Stefanick, M.L., Murray, A.M., Ockene, J., Davatzikos, C., Womens Hlth Initiative Memory, S., 2009. Postmenopausal hormone therapy and regional brain volumes the WHIMS-MRI Study. Neurology 72, 135-142.

Rosene, D., Hoesen, G., 1987. The hippocampal formation of the primate brain. In: Jones, E., Peters, A. (Eds.), Cerebral cortex. Springer, US, pp. 345-456.

Rosenkranz, J.A., Venheim, E.R., Padival, M., 2010. Chronic stress causes amygdala hyperexcitability in rodents. Biol. Psychiatry 67, 1128-1136.

Rubinow, M.J., Drogos, L.L., Juraska, J.M., 2009. Age-related dendritic hypertrophy and sexual dimorphism in rat basolateral amygdala. Neurobiol. Aging 30, 137-146.

Saleem, K.S., Logothetis, N.K., 2006. A Combined MRI and Histology Atlas of the Rhesus Monkey Brain in Stereotaxic Coordinates. Academic Press.

Sallet, J., Mars, R.B., Noonan, M.P., Andersson, J.L., O'Reilly, J.X., Jbabdi, S., Croxson, P.L., Jenkinson, M., Miller, K.L., Rushworth, M.F., 2011. Social network size affects neural circuits in macaques. Science 334, 697-700.

Sanchez, M.M., Hearn, E.F., Do, D., Rilling, J.K., Herndon, J.G., 1998. Differential rearing affects corpus callosum size and cognitive function of rhesus monkeys. Brain Res. 812, 38-49.

Sánchez, M.M., Young, L.J., Plotsky, P.M., Insel, T.R., 2000. Distribution of corticosteroid receptors in the Rhesus brain: relative absence of glucocorticoid receptors in the hippocampal formation. J. Neurosci. 20, 4657-4668.

Seeman, M.V., 1997. Psychopathology in women and men: focus on female hormones Am. J. Psychiatry 154, 1641-1647.

Shansky, R.M., Hamo, C., Hof, P.R., Lou, W., McEwen, B.S., Morrison, J.H., 2010. Estrogen promotes stress sensitivity in a prefrontal cortex-amygdala pathway. Cereb. Cortex 20, 2560-2567.

Shively, C.A., Register, T.C., Clarkson, T.B., 2009. Social stress, visceral obesity, and coronary artery atherosclerosis: product of a primate adaptation. Am. J. Primatol. 71, $742-751$.

Short, S.J., Lubach, G.R., Karasin, A.I., Olsen, C.W., Styner, M., Knickmeyer, R.C., Gilmore, J.H., Coe, C.L., 2010. Maternal influenza infection during pregnancy impacts postnatal brain development in the rhesus monkey. Biol. Psychiatry 67, 965-973.

Shughrue, P.J., Lane, M.V., Merchenthaler, I., 1997. Comparative distribution of estrogen receptor-alpha and -beta mRNA in the rat central nervous system. J. Comp. Neurol. 388, 507-525.

Silk, J.B., 2002. Practice random acts of aggression and senseless acts of intimidation: the logic of status contests in social groups. Evol. Anthropol. Issues News Rev. 11, $221-225$.

Smaers, J.B., Steele, J., Case, C.R., Cowper, A., Amunts, K., Zilles, K., 2011. Primate prefrontal cortex evolution: human brains are the extreme of a lateralized ape trend. Brain Behav. Evol. 77, 67-78.

Spinelli, S., Chefer, S., Suomi, S.J., Higley, J.D., Barr, C.S., Stein, E., 2009. Early-life stress induces long-term morphologic changes in primate brain. Arch. Gen. Psychiatry 66, $658-665$.

Styner, M., Knickmeyer, R., Joshi, S., Coe, C., Short, S.J., Gilmore, J., 2007. Automatic Brain Segmentation in Rhesus Monkeys, Medical imaging. International Society for Optics and Photonics pp. 65122L-65122L-65128.

Toufexis, D., Rivarola, M.A., Lara, H., Viau, V., 2014. Stress and the reproductive axis. J. Neuroendocrinol. 26, 573-586.

Wallace, M., Luine, V., Arellanos, A., Frankfurt, M., 2006. Ovariectomized rats show decreased recognition memory and spine density in the hippocampus and prefrontal cortex. Brain Res. 1126, 176-182.

Wang, J., Vachet, C., Rumple, A., Gouttard, S., Ouziel, C., Perrot, E., Du, G., Huang, X., Gerig, G., Styner, M.A., 2014. Multi-atlas segmentation of subcortical brain structures via the AutoSeg software pipeline. Front. Neuroinform. 8.

Willard, S.L., Daunais, J.B., Cline, J.M., Shively, C.A., 2011. Hippocampal volume in postmenopausal cynomolgus macaques with behavioral depression. Menopause-the Journal of the North American Menopause Society 18, 582-586.

Wilson, M.E., 2016. An introduction to the female Macaque model of social subordination stress. In: Shively, C.A., Wilson, M.E. (Eds.), Social Inequalities in Health in Nonhuman Primates: The Biology of the Gradient. Springer International Publishing, Cham, pp. 9-24.

Wilson, M.E., Gordon, T.P., Collins, D.C., 1982. Variation in ovarian steroids associated with the annual mating period in female rhesus monkeys (Macaca mulatta). Biol. Reprod. 27, 530-539.

Wnuk, A., Korol, D.L., Erickson, K.I., 2012. Estrogens, hormone therapy, and hippocampal volume in postmenopausal women. Maturitas 73, 186-190. 